\title{
Pre-pregnancy body mass index does not impact live birth rate after frozen-thawed euploid embryo transfer: a retrospective cohort study
}

\author{
Xitong Liu ${ }^{1}$, Li Tian ${ }^{1}$, He Cai ${ }^{1}$, Juanzi Shi ${ }^{1}$, and Haiyan Bai ${ }^{1}$ \\ ${ }^{1}$ Affiliation not available
}

May 16, 2021

\begin{abstract}
Objective To assess the effects body mass index (BMI) on live birth rates in patients undergoing in vitro fertilization (IVF) with trophectoderm biopsy and preimplantation genetic testing for aneuploidies (PGT-A) when transferring a single euploid blastocyst. Design Retrospective cohort study. Setting Public fertility center in China. Population 821 women who underwent first cycle of frozen-thawed single euploid blastocyst transfer between 2012 and 2020. Methods Patients were grouped by World Health Organization (WHO) BMI class: underweight $(<18.5, \mathrm{n}=80)$, normal weight $(18.5-24.9, \mathrm{n}=602)$, overweight $(25$-30, $\mathrm{n}=112)$, and obese $([?] 30, \mathrm{n}=27)$. A logistic regression model was used to assess the association between BMI and live birth while adjusting for potential confounders. Main outcome measures Live-birth rate was primary outcome. Results There was no difference in the birth weight, miscarriage, preterm birth, pregnancy complication, type of delivery and fetal gender by BMI category. The clinical pregnancy rate was higher in the overweight and obese groups. In multivariate logistic regression analysis, we fail to demonstrate a statistically significant relationship between BMI and live birth in underweight (adjusted odds ratio [AOR] 0.80; 95\% confidence interval [CI], 0.47-1.35, p=0.402), overweight (AOR 0.85; 95\% CI, 0.54-1.35, p=0.491) or obese (AOR 1.07; 95\% CI, 0.48-2.38, $\mathrm{p}=0.864$ ) patients compared with the normal weight reference group. Conclusion No statistically significant relationship was identified between BMI and live birth in patients undergoing IVF with PGT-A, suggesting that the negative impact of obesity on IVF and clinical outcomes may be related to aneuploidy.
\end{abstract}

Running title: BMI does not impact live birth

Pre-pregnancy body mass index does not impact live birth rate after frozen-thawed euploid embryo transfer: a retrospective cohort study

Xitong Liu, ${ }^{\text {a }}$ Li Tian, ${ }^{\text {a }}$ He Cai, ${ }^{\text {a }}$ Juanzi Shi, ${ }^{\text {a }}$ Haiyan Bai, ${ }^{\text {a }}$

a The Assisted Reproduction Center, Northwest Women's and Children's Hospital, Xi'an, China

Correspondence: Haiyan Bai, The Assisted Reproduction Center, Northwest women's and Children's Hospital, Xi'an, China.

Email: flyindance29578@qq.com

ObjectiveTo assess the effects body mass index (BMI) on live birth rates in patients undergoing in vitro fertilization (IVF) with trophectoderm biopsy and preimplantation genetic testing for aneuploidies (PGT-A) when transferring a single euploid blastocyst.

Design Retrospective cohort study.

Setting Public fertility center in China.

Population 821 women who underwent first cycle of frozen-thawed single euploid blastocyst transfer between 2012 and 2020. 
Methods Patients were grouped by World Health Organization (WHO) BMI class: underweight $(<18.5$, $\mathrm{n}=80$ ), normal weight (18.5-24.9, $\mathrm{n}=602)$, overweight $(25-30, \mathrm{n}=112)$, and obese ([?]30, $\mathrm{n}=27)$. A logistic regression model was used to assess the association between BMI and live birth while adjusting for potential confounders.

Main outcome measures Live-birth rate was primary outcome.

Results There was no difference in the birth weight, miscarriage, preterm birth, pregnancy complication, type of delivery and fetal gender by BMI category. The clinical pregnancy rate was higher in the overweight and obese groups. In multivariate logistic regression analysis, we fail to demonstrate a statistically significant relationship between BMI and live birth in underweight (adjusted odds ratio [AOR] 0.80;95\% confidence interval [CI], 0.47-1.35, $\mathrm{p}=0.402$ ), overweight (AOR 0.85; 95\% CI, 0.54-1.35, $\mathrm{p}=0.491$ ) or obese (AOR 1.07; $95 \%$ CI, 0.48-2.38, $\mathrm{p}=0.864$ ) patients compared with the normal weight reference group.

Conclusion No statistically significant relationship was identified between BMI and live birth in patients undergoing IVF with PGT-A, suggesting that the negative impact of obesity on IVF and clinical outcomes may be related to aneuploidy.

Keywords body mass index (BMI), euploid, live birth rate

Tweetable abstract In women undergoing IVF with PGT-A, BMI does not influence live birth.

\section{Introduction}

As obesity became one of the most important threats to human health, they also contribute to pregnancy complications, including preeclampsia, gestational diabetes mellitus (GDM), preterm delivery, cesarean section and small as well as large for gestational age neonates [1,2]. Additionally, obesity has been associated with adverse reproductive outcomes [3-6].

An increasing number of obese women are receiving in vitro fertilization (IVF). However, the etiology for impaired clinical outcomes in obesity remains unclear. Obesity might influence clinical outcomes by reducing oocyte quality, maturity and endometrial receptivity [7]. One of the major limitations in the evaluation of BMI is that embryo chromosome testing is often not performed. Most of the studies on effects of BMI on IVF focused on the impact of adiposity on ovarian responsiveness during ovarian hyperstimulation. In contrast, the effects of pre-pregnancy BMI on risks of live birth in women undergoing frozen-thawed euploid embryo transfer have not yet been adequately studied.

The objective of this study was to determine whether BMI is associated with live birth in women with euploid embryo transfer. This was performed to determine if euploid embryo from women with different BMI have an altered potential.

\section{Methods}

\section{Study design and patients}

This retrospective cohort study includes 821 women with euploid embryo who underwent frozen-thawed embryo transfer between January 2012 and March 2020 in the Center for Assisted Reproductive Technology of Northwest Women's and Children's Hospital, China. All the patients were followed-up for one year after embryo transfer. This study was approved by the ethics committee of the hospital (number 2019013). Because of the retrospective character of the study, informed consent was waived. Patient data were anonymized. Data was extracted from electronic medical record system. Inclusion criteria were: first transfer cycle of a single frozen-thawed, euploid blastocyst embryo after IVF with preimplantation genetic testing for aneuploidies (PGT-A). Exclusion criteria were multiple FET cycles. Patients were included a single time in the study. BMI was calculated as weight divided by squared height. Women were considered underweight if BMI was $<18.5 \mathrm{~kg} / \mathrm{m}^{2}$, normal weight if $18.5 \mathrm{~kg} / \mathrm{m}^{2}$ [?] BMI $<25 \mathrm{~kg} / \mathrm{m}^{2}$, overweight if $25 \mathrm{~kg} / \mathrm{m}^{2}$ [?] BMI $<30 \mathrm{~kg} / \mathrm{m}^{2}$, and obese if BMI was [?] $30 \mathrm{~kg} / \mathrm{m}^{2}$ according to the most recent BMI classification by the World Health Organization (WHO) [8]. 


\section{IVF procedure}

All the patients received a standardized ovarian stimulation regimen in the PGT-A cycles according to their ovarian function. In brief, recombinant FSH was used for ovarian stimulation in gonadotropin-releasing hormone agonist, gonadotropin-releasing hormone antagonist protocols or others. Oocyte retrieval was performed $36 \mathrm{~h}$ after triggering with hCG.

Insemination of retrieved oocytes was done by intracytoplasmic sperm injection (ICSI). Laser-assisted breaching of the zona pellucida was performed on day 3. The embryos were assessed on day 5 and 6 , and trophectoderm (TE) biopsy was performed. Biopsied TE cells were then stored at -20 for future whole genome amplification (WGA) and next generation sequencing (NGS). After the biopsy, blastocysts were vitrified to be replaced in the subsequent frozen-thawed embryo transfer cycle.

Only cycles where at least one euploid blastocyst was available for transfer were included in this study. In ovulatory patients, embryo transfers were timed after ultrasound confirming ovulation. Anovulatory women were managed with an artificial hormone replacement regime. Pregnancy was confirmed by serum $\beta$-hCG assessment 14 days after embryo transfer.

\section{Definition of clinical outcomes}

Clinical pregnancy was defined as the presence of gestational sac at the 6-8 weeks transvaginal ultrasound. Ectopic pregnancy was defined as gestational sac observed by ultrasound outside the uterine cavity. Miscarriage was defined as spontaneous loss of a clinical pregnancy before 24 completed weeks of gestational age, in which the embryo(s) or fetus(es) is/are nonviable and is/are not spontaneously absorbed or expelled from the uterus. Preterm birth was defined as a birth that takes place after 24 weeks and before 37 completed weeks of gestational age. Live birth was defined as the delivery of at least one live-born baby beyond 24 weeks of gestational age. Live birth rate was defined as live deliveries (at least one live birth) per women after embryo transfer.

\section{Statistical analysis}

Data are presented as mean and standard deviation (continuous variables) or counts and proportions (categorical variables). One-way analysis of variance was used for continuous variables, and the Pearson's $\chi^{2}$ test was used for categorical variables with Fisher's exact test when necessary. We performed logistic regression to explore the effects of BMI on live birth after accounting for the following potential confounders: female age, infertility duration, endometrium (EM) thickness, no. of oocyte retrieved, infertility type, protocol in fresh cycle (agonist, antagonist, other); EM protocol (natural cycle, HRT, GnRHa+HRT). We calculated crude odds ratios (OR) and adjusted ORs (aOR) with 95\% confidence interval (CI). Interaction and stratified analyses were conducted according to confounders. Data were analyzed with the use of the statistical packages R (The R Foundation; http://www.r-project.org;version 3.4.3) and Empower (R) (www.empowerstates.com, $\mathrm{X} \& \mathrm{Y}$ solutions, inc. Boston, Massachusetts). The level of significance was set at $p<0.05$.

\section{Results}

Among 821 cycles that were started between 2012 and 2020 and in which a single frozen-thawed, euploid blastocyst embryo transfer strategy was applied, most women were of normal weight (BMI, $18.5-25 \mathrm{~kg} / \mathrm{m}^{2}$, $\mathrm{n}=602)$. A total of 80 of the cycles involved underweight women with a low BMI (BMI $\left.<18.5 \mathrm{~kg} / \mathrm{m}^{2}\right), 112$ of the cycles involved overweight women (BMI, $25-30 \mathrm{~kg} / \mathrm{m}^{2}$ ), and 27 of the cycles involved obese women (BMI [?] $30 \mathrm{~kg} / \mathrm{m}^{2}$ ). The baseline characteristics of participants are presented in Table 1 . There was a significant difference in endometrial thickness in FET between groups, with overweight women more likely to have thicker endometrium. Infertility type and protocol in fresh cycle differed significantly across the four groups, with obese women more prevalent with more primary infertility and agonist protocol. The overweight women had a higher rate of clinical pregnancy rate. However, there were no other specific differences in fertility parameters between different BMI categories.

Univariate analysis was performed to evaluate each variable's effect on live birth rate (Table 2). Female age 
in fresh cycle was negatively associated with live birth, while agonist protocol was positively associated with live birth. Interaction and stratified analyses were shown in Table S1. There were no significant interactions in any of the subgroups ( $\mathrm{p}>0.05$ for all comparisons).

Figure 1 shows the spline-transformed association of BMI with live birth, and BMI tended to be linearly associated with live birth. Figure S1 shows NGS PGT cycle outcomes from the year 2012 to 2020. Figure S2 shows survival rates from 2012 to 2020 . Multivariate logistic regression failed to show a statistically significant relationship between BMI and live birth in underweight (BMI $<18.5 \mathrm{~kg} / \mathrm{m}^{2}$; adjusted odds ratio [AOR] 0.80; 95\% confidence interval [CI], 0.47-1.35, $\mathrm{p}=0.402$ ), overweight (BMI 25-30 kg/m²: AOR 0.85; 95\% CI, 0.54-1.35, $\mathrm{p}=0.491$ ), and obese patients (BMI[?] $30 \mathrm{~kg} / \mathrm{m}^{2}:$ AOR 1.07; 95\% CI, 0.48-2.38, $\mathrm{p}=0.864$ ) compared with the normal weight reference group (Table 3 ).

\section{Discussion}

\section{Main findings}

In this retrospective cohort study of 821 women undergoing IVF with euploid blastocyst embryo transfer, we showed that live birth was not significantly different between obese and normal-weight women.

The prevalence of obesity continues to rise worldwide. The negative consequences of obesity are identifiable in every organ system. Obesity is a known risk factor for subfertility, miscarriage, feto-maternal complications and long-term risks in adult life [9]. Obese women are at particularly high risk for adverse pregnancy outcomes. Up till now, the relationship between obesity and negative reproductive outcomes is clear, but the mechanism by which obesity affects fertility remains unclear. Data from large trials have demonstrated fewer normally fertilized oocytes, lower pregnancy and live birth rates in obese women [10]. We believe that our study adds important information to the existing literature on pre-pregnancy BMI. A large cohort study of 152,500 cycles similarly reported significantly higher odds of cycle cancellation, pregnancy failures with overweight women [11]. A systematic review and meta-analysis found decreased pregnancy rates, increased gonadotropins and a higher miscarriage rate in obese and overweight women. These differences are evidence even at a BMI[?] $25 \mathrm{~kg} / \mathrm{m}^{2}[12]$.

Our study differs from previous studies on obesity and clinical outcomes in three ways. First, most studies dealt with embryo transfer without PGT-A. Theorizing that oocytes from obese patients are subject to alterations in normal mitotic checkpoints leading to aneuploidy [13]. Therefore, they cannot accurately elucidate the effects of BMI on clinical outcomes in obese women. Second, many studies dealt with obesity as a categorical variable. In contrast, in our study, BMI was also analyzed as a continuous variable that allowed a subtle increase in live birth. Third, some studies compared clinical outcomes in fresh cycles. One possible mechanism suggested that endometrial advancement induced by ovarian hyperstimulation in a fresh embryo transfer cycle, resulting in embryo-endometrium asynchrony. Therefore, our study focused on frozen embryo transfer cycles, which eliminate the negative effect of controlled ovarian stimulation.

\section{Strengths and limitations}

Our present work has some strength of note. Firstly, we adjusted more variables to ensure that the result was more reliable. Secondly, proper statistical methods and sensitivity analysis ensure structural stability. Thirdly, the study included solely women underwent PGT-A to cleanly analyze the impact of BMI on live birth. Last but not least, we only included women in the first cycle of FET, thus avoiding bias from multiple cycles.

There are some limitations to this study, such as its retrospective nature, which reduces its direct application to clinical practice. The study question would best be addressed by a multicenter, prospective trial. Our study is also limited by the relatively small proportion of overweight and obese women in our cohort.

\section{Interpretation}

We found no association between obesity and live birth when transferring a single euploid blastocyst embryo. Our results suggest that the negative impact of being overweight or obese may be related to embryonic 
aneuploidy. A prior meta-analysis showed that a high BMI did not affect the IVF clinical outcomes in donor oocyte recipients, which suggested that oocyte quality may be the most important factor in obese women [14]. Previous studies suggested metabolic alterations in the serum are reflected in the follicular fluid and that some of these alterations may affect oocyte quality with higher BMI both in vitro and in vivo [15-17]. In addition, one prospective cohort study showed that elevated follicular follicular free fatty acid (FFA) were associated with poor cumulus oocyte complex (COC) morphology [18]. Moreover, severe obesity is also associated with a greater prevalence of spindle anomalies and non-aligned chromosomes in failed fertilized oocytes [19].

Embryo aneuploidy, which is widely known to be the most important factor for negative outcomes, was not given enough attention in previous studies when comparing the live birth rate between obese and normalweight women. Obesity has been reported to alter the early embryo metabolomic signature [20], raising the possibility of epigenetic mediated impairment of clinical outcomes.

\section{Conclusion}

Our results demonstrated no relationship between BMI and live birth in women undergoing single euploid blastocyst embryo with PGT-A, suggesting that the detrimental effect of an elevated BMI on pregnancy and IVF outcomes may be the result of genomic, or an unspecified alternative etiology. While the findings of this study are valuable in contributing towards current understanding of the relationship between BMI and pregnancy outcome, further research needs to be undertaken before the results can be extrapolated to the general population.

Disclosure of interests

None declared.

Contribution to authorship

XL designed the study. LT and HC conducted data collection, analysis and writing the manuscript. JS and HB conceived and designed the study.

\section{Details of ethics approval}

This study was approved by the ethics committee of the hospital (number 2019013).

\section{Funding}

Supported by National Natural Science Foundation of China (No. 81771657 http://www.nsfc.gov.cn/) and General Projects of Social Development in Shaanxi Province(No. 2018SF-260).

\section{Acknowledgements}

The authors thank the staff from Northwest Women's and Children's Hospital for their assistance with the data collection.

\section{References}

1. Lu GC, Rouse DJ, DuBard M, Cliver S, Kimberlin D, Hauth JC. The effect of the increasing prevalence of maternal obesity on perinatal morbidity. American journal of obstetrics and gynecology. 2001; 185(4): $845-9$.

2. Rajasingam D, Seed PT, Briley AL, Shennan AH, Poston L. A prospective study of pregnancy outcome and biomarkers of oxidative stress in nulliparous obese women. American journal of obstetrics and gynecology. 2009; 200(4): 395.e1-9.

3. Metwally M, Ong KJ, Ledger WL, Li TC. Does high body mass index increase the risk of miscarriage after spontaneous and assisted conception? A meta-analysis of the evidence. Fertility and sterility. 2008; 90(3): 714-26. 
4. Boots CE, Bernardi LA, Stephenson MD. Frequency of euploid miscarriage is increased in obese women with recurrent early pregnancy loss. Fertility and sterility. 2014; 102(2): 455-9.

5. Metwally M, Saravelos SH, Ledger WL, Li TC. Body mass index and risk of miscarriage in women with recurrent miscarriage. Fertility and sterility. 2010; 94(1): 290-5.

6. Lo W, Rai R, Hameed A, Brailsford SR, Al-Ghamdi AA, Regan L. The effect of body mass index on the outcome of pregnancy in women with recurrent miscarriage. Journal of family \& community medicine. 2012; 19(3): 167-71.

7. Zhang JJ, Feret M, Chang L, Yang M, Merhi Z. Obesity adversely impacts the number and maturity of oocytes in conventional IVF not in minimal stimulation IVF. Gynecological endocrinology : the official journal of the International Society of Gynecological Endocrinology. 2015; 31(5): 409-13.

8. WHO Expert Consultation. Appropriate body-mass index for Asian populations and its implications for policy and intervention strategies. Lancet (London, England). 2004; 363(9403): 157-63.

9. Triunfo S, Lanzone A. Impact of overweight and obesity on obstetric outcomes. Journal of endocrinological investigation. 2014; 37(4): 323-9.

10. Shah DK, Missmer SA, Berry KF, Racowsky C, Ginsburg ES. Effect of obesity on oocyte and embryo quality in women undergoing in vitro fertilization. Obstetrics and gynecology. 2011; 118(1): 63-70.

11. Luke B, Brown MB, Missmer SA, Bukulmez O, Leach R, Stern JE. The effect of increasing obesity on the response to and outcome of assisted reproductive technology: a national study. Fertility and sterility. 2011; 96(4): 820-5.

12. Maheshwari A, Stofberg L, Bhattacharya S. Effect of overweight and obesity on assisted reproductive technology-a systematic review. Human reproduction update. 2007; 13(5): 433-44.

13. Goldman KN, Hodes-Wertz B, McCulloh DH, Flom JD, Grifo JA. Association of body mass index with embryonic aneuploidy. Fertility and sterility. 2015; 103(3): 744-8.

14. Jungheim ES, Schon SB, Schulte MB, DeUgarte DA, Fowler SA, Tuuli MG. IVF outcomes in obese donor oocyte recipients: a systematic review and meta-analysis. Human reproduction (Oxford, England). 2013; 28(10): 2720-7.

15. Valckx SD, De Pauw I, De Neubourg D, Inion I, Berth M, Fransen E et al. BMI-related metabolic composition of the follicular fluid of women undergoing assisted reproductive treatment and the consequences for oocyte and embryo quality. Human reproduction (Oxford, England). 2012; 27(12): 3531-9.

16. Leroy JL, Vanholder T, Delanghe JR, Opsomer G, Van Soom A, Bols PE et al. Metabolic changes in follicular fluid of the dominant follicle in high-yielding dairy cows early post partum. Theriogenology. 2004; 62(6): 1131-43.

17. Leroy JL, Vanholder T, Mateusen B, Christophe A, Opsomer G, de Kruif A et al. Non-esterified fatty acids in follicular fluid of dairy cows and their effect on developmental capacity of bovine oocytes in vitro. Reproduction (Cambridge, England). 2005; 130(4): 485-95.

18. Jungheim ES, Macones GA, Odem RR, Patterson BW, Lanzendorf SE, Ratts VS et al. Associations between free fatty acids, cumulus oocyte complex morphology and ovarian function during in vitro fertilization. Fertility and sterility. 2011; 95(6): 1970-4.

19. Machtinger R, Combelles CM, Missmer SA, Correia KF, Fox JH, Racowsky C. The association between severe obesity and characteristics of failed fertilized oocytes. Human reproduction (Oxford, England). 2012; 27(11): 3198-207.

20. Bellver J, Rossal LP, Bosch E, Zúñiga A, Corona JT, Meléndez F et al. Obesity and the risk of spontaneous abortion after oocyte donation. Fertility and sterility. 2003; 79(5): 1136-40. 


\section{Hosted file}

table.pdf available at https://authorea.com/users/373747/articles/522180-pre-pregnancy-bodymass-index-does-not-impact-live-birth-rate-after-frozen-thawed-euploid-embryo-transfera-retrospective-cohort-study
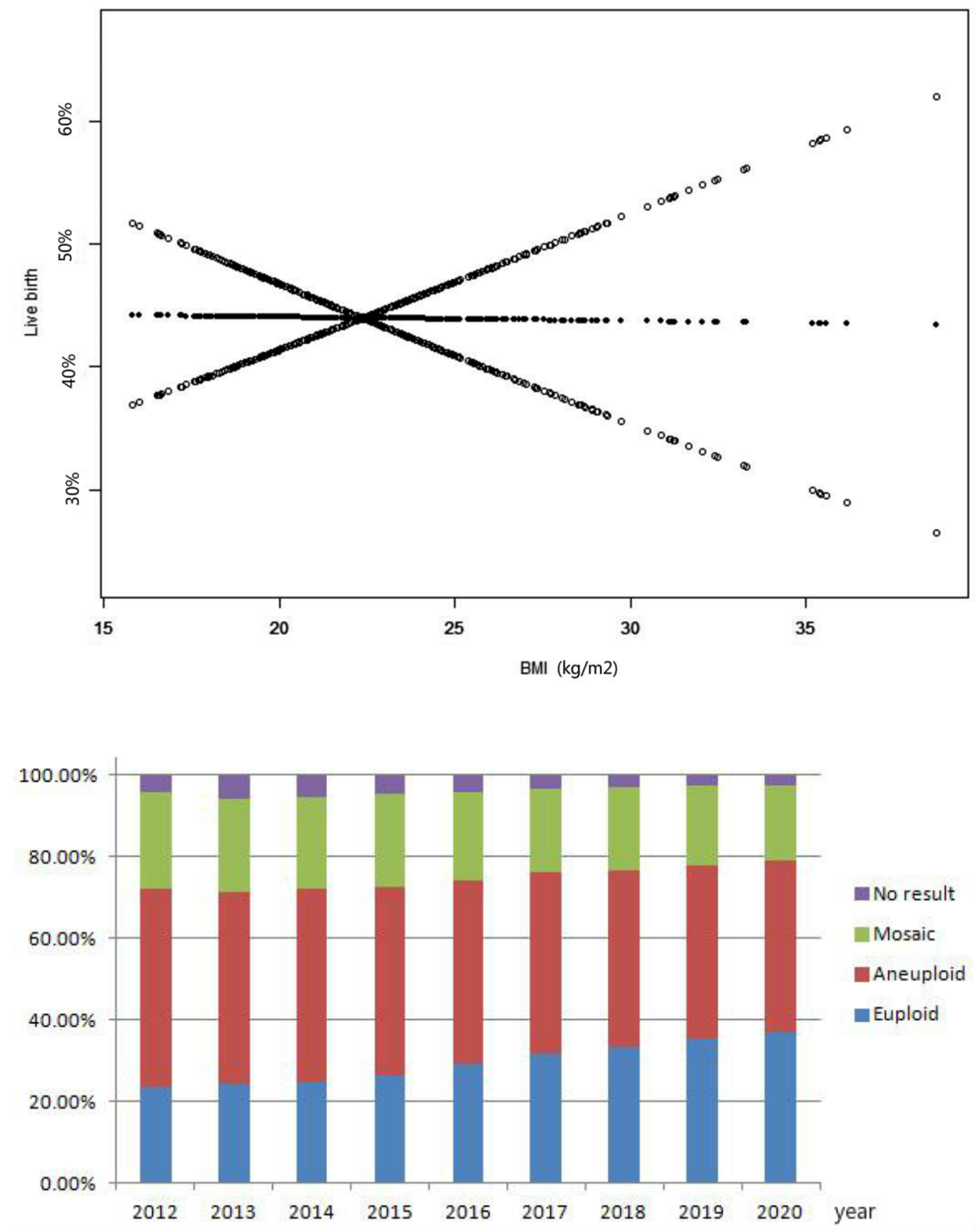


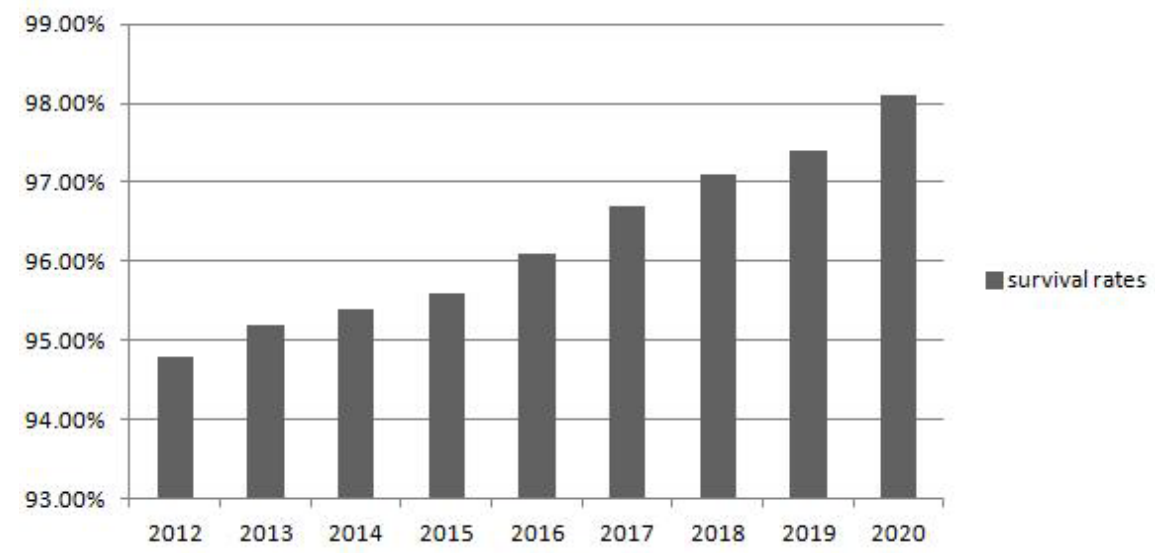

